\title{
Modelling the propagation pathway of street-traffic noise: practical comparison of German guidelines and real-world measurements
}

\author{
E. Wetzel ${ }^{\mathrm{a}}$, J. Nicolas ${ }^{\mathrm{b}}$, Ph. Andre ${ }^{\mathrm{b}}$, J.-J. Boreux ${ }^{\mathrm{b}}$ \\ ${ }^{a}$ Wolfel Meßsysteme Software GmbH \& Co, Hochberg, Germany. \\ ${ }^{b}$ Fondation Universitaire Luxembourgeoise, Arlon, Belgium
}

\begin{abstract}
:
In Germany, several guidelines were developed to model the noise propagation pathway. The study compares the guidelines DIN 18005, RLS-90 and VDI 2714, all of which provide some kind of sound object line source suited to model street traffic noise. Differences between those guidelines are explained, and their effect on practical calculations is shown in a real-world situation, National Road N4, in Arlon, Belgium. Calculated results are compared to measurements made on critical points along the road. The paper emphasises the understanding of the inner workings of models. In order to avoid differences in calculated results due to software design methods applied, all calculations are made using one single commercially available simulation program. Additionally, this allows for a test of guideline sensitivity to changes in input parameters.
\end{abstract}

\section{Introduction}

Increased use of noise prediction software implementing mathematical models describing the noise propagation pathway outdoors led to a discussion on model accuracy and sensitivity. Previous model comparisons either intend to validate an original method against commercial programs [1,2] or concentrate on model intercomparison, neglecting both comparison to real-world measurements [3,4] and the model's intrinsic sensitivity to user specified input parameters. In addition, the user's experience and understanding of the model and the software used is generally left out, as are the influence of design decisions made by the software developer, and manipulation errors. The present paper shows both model sensitivity to input parameters (vehicle type and speed, road surfacing and slope, ...) and coherence of results with field measurements.

Measurement and modelling is done for traffic noise from a typical four lane road. Modelling practice in most European countries and choices for ISO 9613-2 show preferences for semi-empirical models. Therefore and to avoid questionable comparisons physical models were not used and focus is on classical semi-empirical noise models. To clear out all disturbing external factors, one single commercially available program IMMI 4.0 [10] was used and operated by the one single user, who also conducted all real-world measurements. The configuration of the software used for the present study relies on calculation guidelines following German standards: DIN 18005 (1987) [7], VDI 2714 (1988) [8] and RLS-90 (1990) [9].

\section{Materials and methods}

\subsection{Model description}

Classical semi-empirical methods rely on the following basic equation:

$L_{i=} L_{e}+D$

with

$L_{i}$ the resulting sound level at the receiving point,

$L_{e}$ the noise emission level, and

$D$ the sum of geometrical divergence, air absorption, ground effect, screening, reflection and miscellaneous other effects.

In semi-empirical models a road is divided into point sources, each of which is both stationary and continuous. Each point's sound emission level is relative to the length of the line segment it represents. The number of point sound sources required to properly represent a line source is determined by the distance criterion

$1<=\beta s$

with 
1 the maximum length of each line segment,

$\beta$ a constant $<1$,

$s$ the distance from the centre of the segment to the receiving point. Otherwise stated, the number of required point sound sources increases with decreasing source to receiver distance, $\beta$ may be different for each guideline. In addition, calculation of pathway difference due to screening obstacles can significantly vary as is shown in the following table (Table 1).

\subsection{Test case}

The guidelines considered in this study provide some kind of sound object line source suited to model street traffic noise.

Table 1: Coefficient $\beta$ and differences in pathlength for different guidelines

\begin{tabular}{llll}
\hline Guideline & DIN 18005 & RLS 90 & VDI 2714 \\
\hline$\beta$ & 0.7 & 0.5 & 0.5 \\
Pathway & $\begin{array}{l}\text { Shortest pathway } \\
\text { difference }\end{array}$ & $\begin{array}{l}\text { Pathway atop the } \\
\text { around the screen }\end{array}$ & $\begin{array}{l}\text { Both pathways atop } \\
\text { and around the } \\
\text { screen only }\end{array}$ \\
\end{tabular}

Namely, the paper deals with two ways of modeling a road in the program IMMI: The first method is the typical way of algorithms that simulate road traffic by means of a line sound source and calculate overall equivalent sound level from traffic characteristics. This is the case for DIN 18005 with road traffic source STRa that models the traffic flow on a lane by a line sound source located $0.5 \mathrm{~m}$ above the centre of the lane, and for RLS 90, with road traffic source STRb that simulates a road with multiple tracks by assuming one line source at $0.5 \mathrm{~m}$ above the middle of each of the two outer tracks. The equivalent sound level is then computed as the energetic sum of the sources. Both models calculate emission levels from relevant traffic density and associated portion of trucks, vehicle speed and road surfacing. The second method uses frequency distribution on sound propagation pathway, by means of a general-purpose line sound source from VDI 2714 guideline (noted LIQc).

All these three ways of simulating street traffic noise are applied on a real-world situation, national road N4, passing straight in front of the Fondation Universitaire Luxembourgeoise, in Arlon, in the south of Belgium. The road is a $15 \mathrm{~m}$ wide and has four lanes to account for high traffic load. Development is unevenly distributed on both sides of the road consisting of a supermarket, some buildings and several dwellings. A $825 \mathrm{~m}$ straight portion of the road is digitised, longitudinally in the middle of a rectangular $900 \mathrm{x} 500 \mathrm{~m}$ work area.

In order to be able to compare simulated results to measurements, all buildings, walls and copses are digitised, as are terrain levels. To reduce the number of influence parameters, it is assumed that the N4 road is the one and only sound source, which is justified as other sources can be neglected with respect to traffic noise at rush hour.

The present paper does neither intend to compare absolute noise levels computed by the various guidelines, nor try to prove comparability of computer simulation and measurements. It rather emphasises the understanding of the inner-working of models, and it compares relative behaviours only: sound equivalent level at $25 \mathrm{~m}$ from road axis is ensured to be equal for all line source types in free propagation conditions.

Average altitude is $405 \mathrm{~m}$. At the centre of the work area, terrain is rather flat, growing up north to $415 \mathrm{~m} \mathrm{level.}$ Ground exhibits a pronounced depression in the centre-east.

Standard configuration chosen for the road is 3000 vehicles/h, with $20 \%$ trucks, the surface is a smooth mastic asphalt and the allowed maximum speed is $80 \mathrm{~km} / \mathrm{h}$.

Roads or line sources are idealised by means of point sources: automatic generation of $10 \mathrm{~m}$ line sections is proposed.

Sound level is calculated for both single receiving points and a square grid of receivers with $10 \mathrm{~m}$ mesh side length. 


\subsection{Noise measurement}

Field measurements are performed at $1.2 \mathrm{~m}$ above ground during rush hour around 5 p.m. using a tripod mounted Brüel and Kjaer, type 2231 equipped with a condenser microphone adapted for free-field conditions and configured for A-weighted frequency response and fast sampling rate.

The equivalent continuous sound level, $L_{\mathrm{eq}}$, is integrated over $5 \mathrm{~min}$, which is considered as sufficient to average the fluctuating noise from traffic during peak period. Vehicles are counted during each measurement period.

\section{Results}

\subsection{Comparison of algorithms}

Fig. 1 shows isolines from $50 \mathrm{~dB}(\mathrm{~A})$ to $75 \mathrm{~dB}(\mathrm{~A})$, resulting from a simulation with STRa line source type from guideline DIN 18005, using previously defined standard conditions. That case will be considered as the base case. Isolines are overlaid on top of the situation plan.

Attenuation by buildings is obvious, mainly in the vicinity of the supermarket, where isophones are very close to one another. When approaching free propagation conditions, as is the case in the centre of the work area, isophones are more evenly distributed with linear attenuation of $3 \mathrm{~dB}(\mathrm{~A})$ per distance doubling.

Ground depression at the centre right part of the work area undoubtedly acts as attenuating element, demonstrating the outstanding importance of topography in noise studies.

On the other hand the influence of copse on the propagation pathway is small: actually, the relevant damping factor is only some $5 \mathrm{~dB}(\mathrm{~A})$ per $100 \mathrm{~m}$.

Relatively small divergence among algorithms tested can be observed: sound level difference between any two calculation methods never exceeds $5 \mathrm{~dB}(\mathrm{~A})$ throughout the whole work area. Definitely, most discrepancies can be attributed to pathlength difference calculation handled differently by each particular guideline : there are sensible differences between DIN 18005 and RLS 90, whereas results from VDI 2714 completely differ. Hatched portion of Fig. 2 represents the area where the immission sound level calculated with STRa (DIN 18005) exceeds the one calculated with STRb (RLS 90). In addition, terrain level contour lines are shown in Fig. 2.

A careful observation of immission levels in connection with the relief of the work area shows that, with STRa algorithm, noise propagates better through flat or rising ground than through depressions. However, STRb line sources from RLS 90 guideline seem to better account for sound diffraction on crests, in a way that noise propagates farther down the ground depression.

Besides specific features of formulas used to calculate attenuation for each guideline, the sound propagation is also influenced by the frequency distribution of the noise source.

Spectral composition by octave levels of typical traffic noise exhibits a regular decrease from 100 to $8000 \mathrm{~Hz}$ [11]: high frequencies are less represented than lower ones. 
Fig. 1. Isophones simulated by STRa algorithm, from guideline DIN 18005.

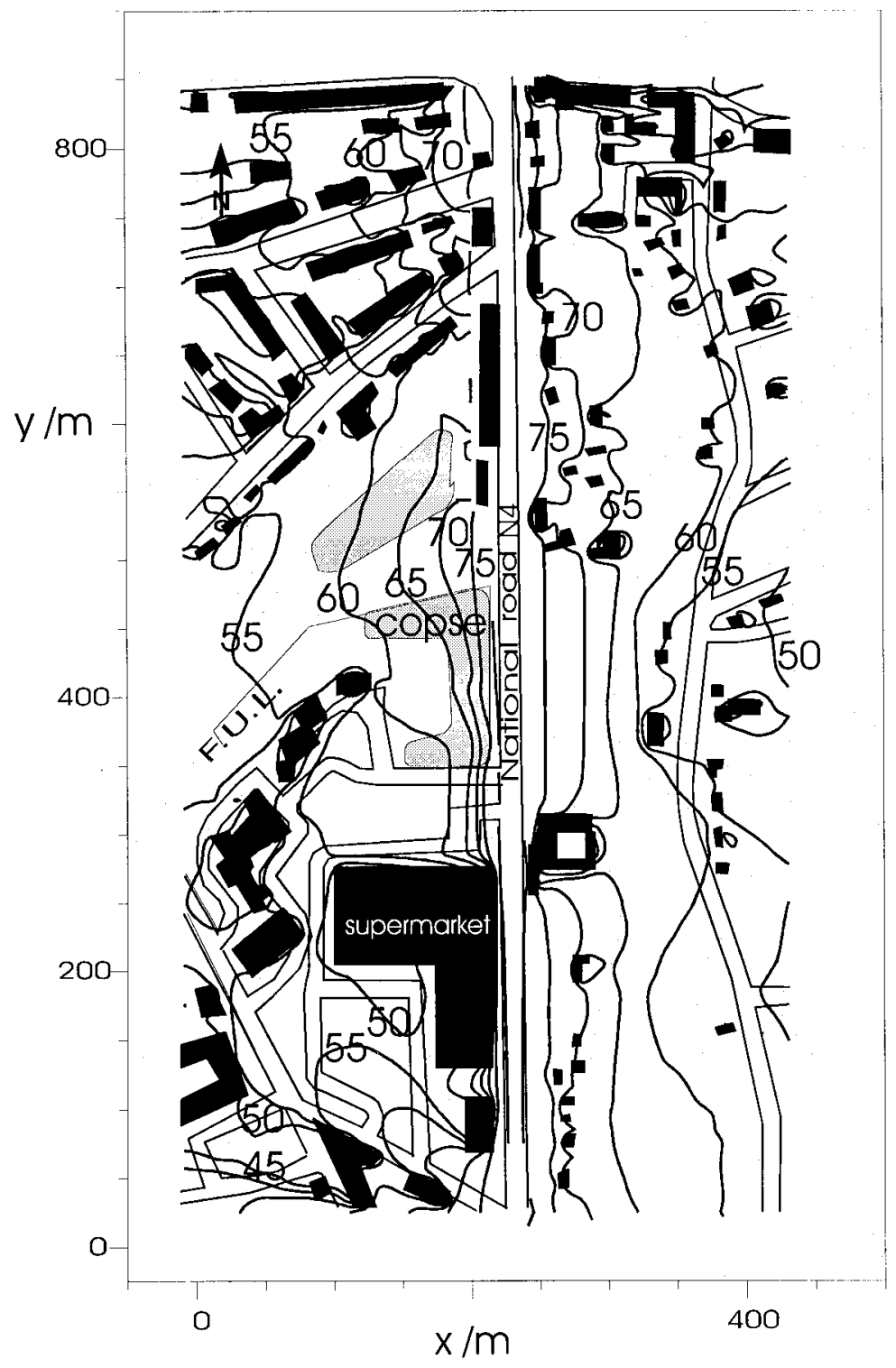

The general-purpose line source LIQc from VDI 2714 guideline allows the user to enter its own frequency distribution.

We have tested, with equivalent global sound levels, both a uniform emission distribution and a distribution favouring sharp sound in place of bass tones. It is clearly observed that the more the sound is sharp, the less far it propagates, chiefly through ground depressions. 
Fig. 2. Immission sound levels calculated with two "line source" objects suited to model street traffic noise. Hatched portion represents the field area where the first one (STRa) exceeds the second one (STRb).

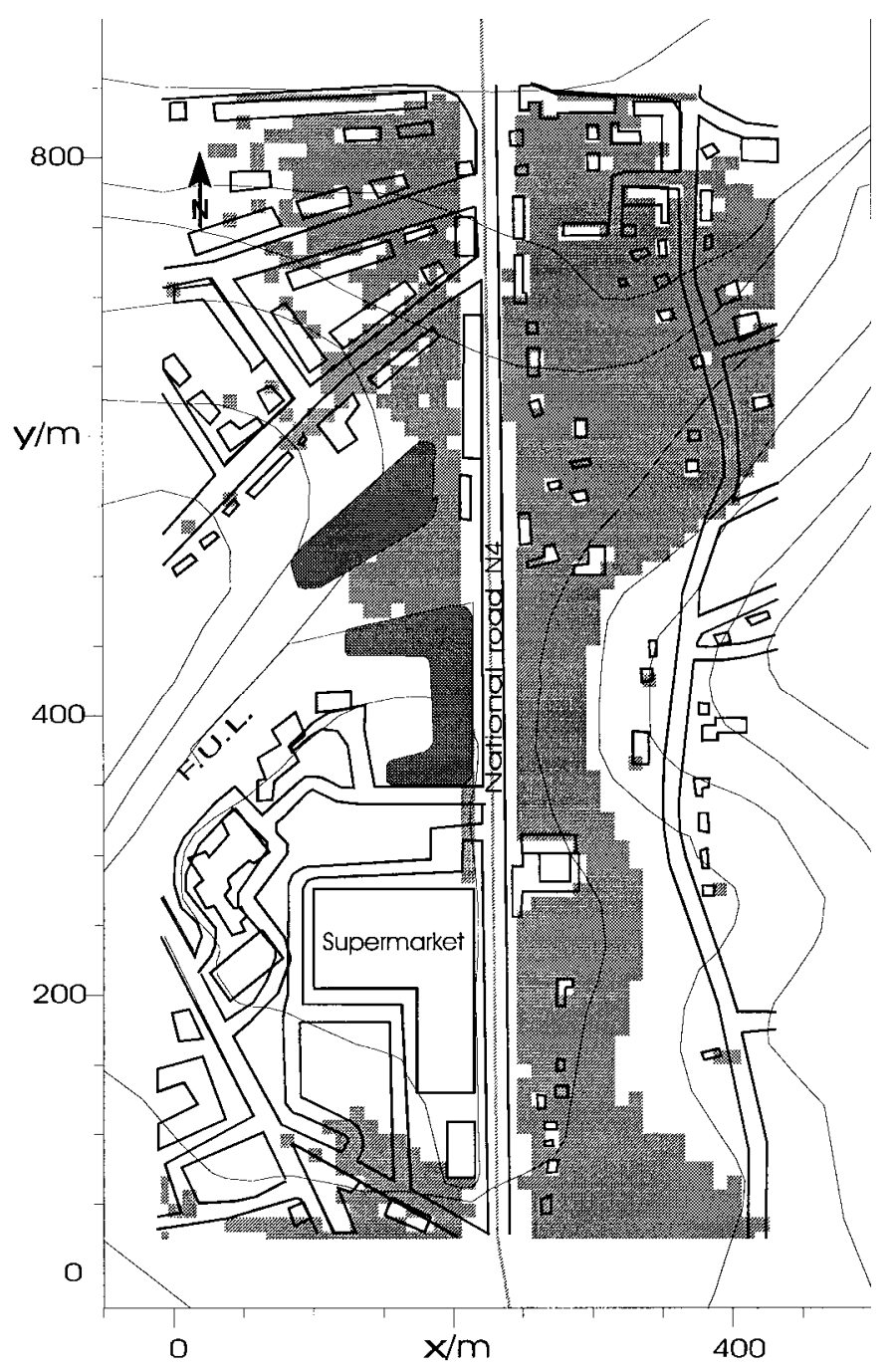

\subsection{Sensitivity analysis}

It is difficult to determine the precision required by the program or the underlying model for estimated or even measured values. Actually, some, like absorption coefficient for structured reflecting walls, can only be estimated. Others, like building heights, are not easy to measure. Finally, for the sake of quick calculations, it can be desired to simplify the data set without introducing additional errors. Therefore sensitivity analysis of calculated immission levels to input parameters is performed.

It was already mentioned that guidelines differ mainly by the formula used to calculate attenuation effects of barriers. The first parameters studied are thus the height and the surface structure of the buildings walls. Here, only one building is modified: the supermarket. The influence of its walls is estimated for three receiving points: a first one located behind the building with respect to the sound source ( $\mathrm{x}=60 \mathrm{~m}, y=100 \mathrm{~m})$, a second one located opposite the supermarket, on the other side of the road $(x=340 \mathrm{~m}, \mathrm{y}=200 \mathrm{~m})$, and a third one located at $400 \mathrm{~m}$ away from the building, to the north $(x=90 \mathrm{~m}, y=500 \mathrm{~m})$.

Only the first receiving point is affected by the modification of the height of supermarket walls. Fig. 3 shows the relative sound level attenuation due to the building height increase, from 0 up to $15 \mathrm{~m}$ for that particular receiver.

The above figures clearly show the suspected differences between guidelines: VDI 2714 (LIQc) calculates the highest attenuation closely followed by RLS 90 (STRb, LIQb), and a distant DIN 18005 (STRa) that seems almost insensitive to building height increase. Here, attention should be brought to another effect: sound reflection on the supermarket wall turned to the street do not affect the receiver located on the other side of the 
road, at about $120 \mathrm{~m}$ from the wall. Varying the reflectivity of the wall surface from $100 \%$ reflectivity to highly absorbing generates an appreciable effect only in the close vicinity of the building.

Parameters that have direct impact on noise emission levels, like vehicle speed, traffic density and the percentage of trucks affect the total absolute immission sound level rather than the transmission pathway. For example, changing the portion of trucks from 20 to $100 \%$ causes a uniform $6 \mathrm{~dB}(\mathrm{~A})$ increase of sound immission level and reducing traffic density from 3000 to 1500 vehicles/h results in a uniform $3 \mathrm{~dB}(\mathrm{~A})$ attenuation.

Fig. 3. Relative sound level attenuation when changing the height of supermarket walls.

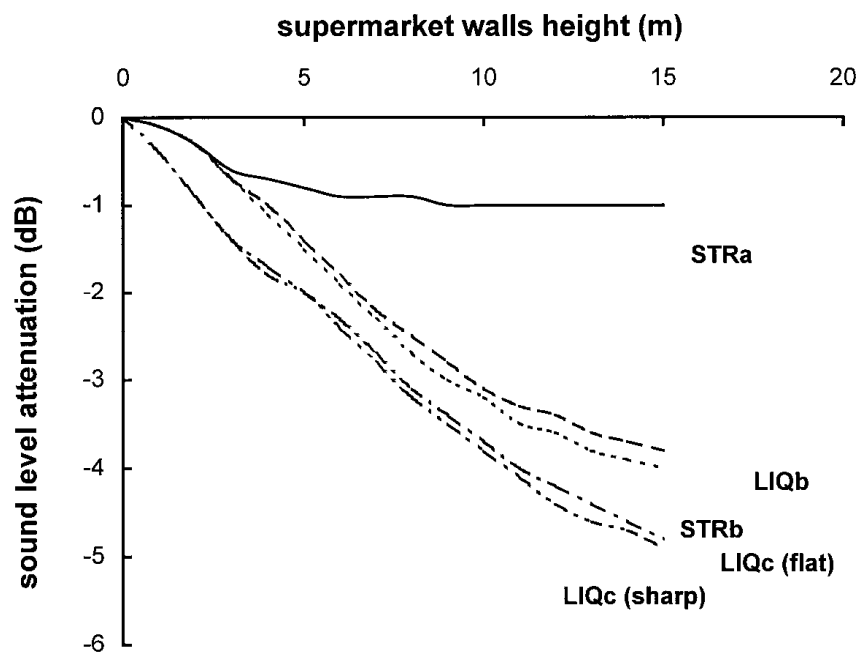

\subsection{Comparison with measurements}

Considering the latter, measurements may be performed even when relevant traffic characteristics do not exactly match those specified in the model. Vehicle speed is supposed to remain constant during one measurement period, but the average values of traffic density and portion of trucks are recorded each time and are allowed to vary from one measurement period to another. Subsequently, every equivalent noise level measurement can be normalised to the same basic conditions, i.e. 3000 vehicles per hour and $20 \%$ of trucks.

To start with, nine measurements were performed in quasi free field propagation conditions, placing the sound level meter on the meadow located in the centre of the work area, west of the road $(y=500 \mathrm{~m})$. Fig. 4 shows the relative noise attenuation for the various algorithms and for the measurements when moving away from the roadside. Fig. 5 shows that on the other side of the road, where ground drops by $10 \mathrm{~m}$ per $100 \mathrm{~m}$ distance, similar noise level attenuation can be found.

Due to diffraction on the ground depression edges, all algorithms generate attenuation curves which are much more undulated than the smooth one in Fig. 4. However, all of them tend to overestimate real values: measured levels decrease systematically faster than the model results, when moving away from the roadside. Moreover, below a certain distance (about $50 \mathrm{~m}$ in this case), STRb algorithm fits the measured points better than any other one; but farther away STRa is closest to measured levels. One may conclude that RLS 90 guideline (STRb, LIQb) overestimates the sound diffraction effect on crests.

In order to judge the ability of equations to handle the attenuation and the reflection effects of barriers, the model results are also compared to measured levels for some receiving points located just behind buildings. Although it is awkward to compare absolute immission level values, the various measurements are normalised in such manner that emission level were similar to that used in simulation. Results show that for receiving points located behind buildings, measured values are always from 2 to $4 \mathrm{~dB}(\mathrm{~A})$ below simulated ones. The methods used by the various guidelines to calculate the sound transmission through obstacles seem thus slightly overestimating the sound level, especially DIN 18005 guideline (STRa) which give rise to the highest immission values behind 
buildings. Finally, the influence of traffic lights on the sound level is tested. Indeed, at the point $(\mathrm{x}=230 \mathrm{~m}, y=$ $25 \mathrm{~m}$ ), on the south of our work area, the main road crosses another one and the two roads are con nected to traffic lights. In the model, traffic lights are simply considered as an additional single sound source, which, in our case, adds $3 \mathrm{~dB}(\mathrm{~A})$ to the emission level of the road at this point. Some measurements performed in the vicinity of the traffic lights show, on the contrary, a decrease of the sound level with respect to other points at the roadside.

As long as this kind of situation is considered (crossing of a high traffic road with a low traffic one), the decrease of sound level due to vehicles slowing down when approaching the traffic lights seems thus prevailing on the noise increase due to cars moving off after the stop.

Fig. 4. Noise attenuation in free field conditions when moving away from the sound source. Comparison model/measurements.

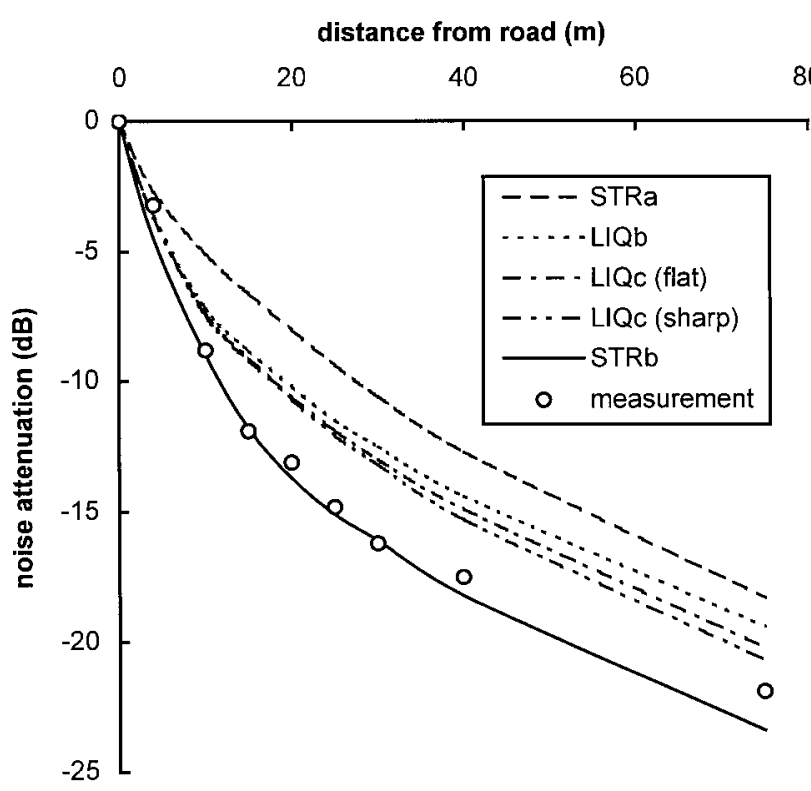

Fig. 5. Noise attenuation when moving away from the road in a ground depression. Comparison model/ measurements.

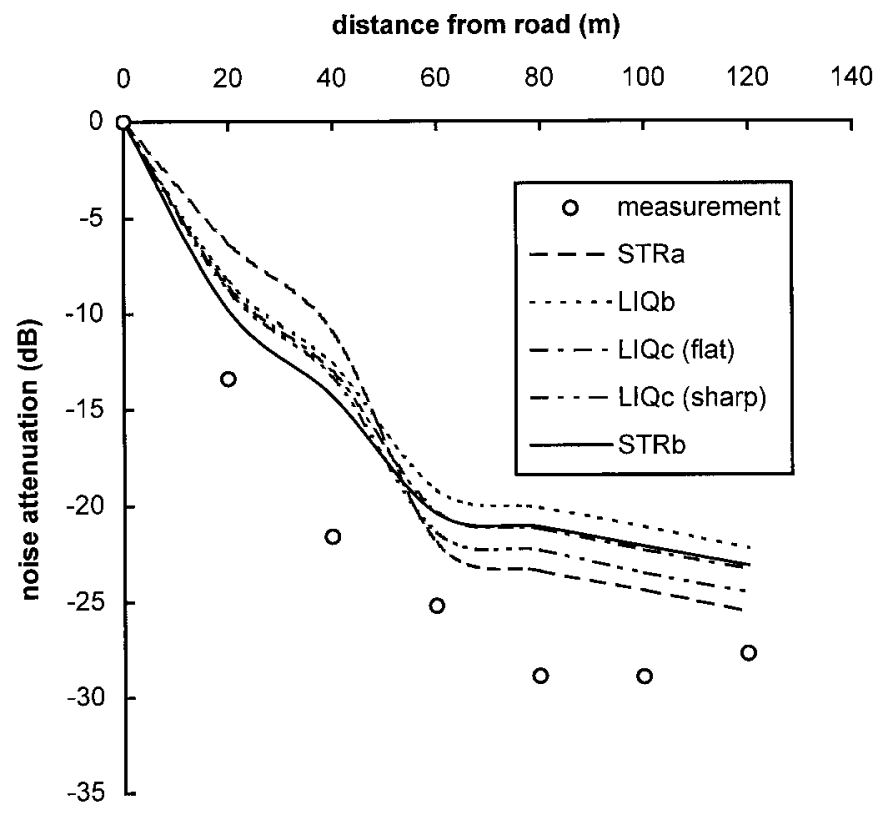




\section{Conclusions}

The comparison of the three German guidelines and the real-world monitoring for the test case of national road N4 in Arlon shows that street objects, as defmed by DIN 18005 and RLS 90 guidelines are well suited to study the noise in the vicinity of a road.

Regarding input parameters of all models, special care should be taken to describe the terrain in the work-area as accurate as possible. The frequency distribution of the sound source is also of paramount importance.

Building and barrier height and width have a general influence on the sound propagation pathway. Although that influence is obvious when analysing real-world measurements, DIN 18005 guideline does not attach sufficiently importance to it. Other guidelines (RLS 90 and VDI 2714) account better for obstacle influence, but calculated attenuation never reaches the measured one.

As for surface structure of walls, its modification generates only slight effect on sound immission level, except for the immediate vicinity of the buildings.

As a matter of fact, RLS 90 guideline is closer to reality than other ones, as long as similar test cases are considered. However, it overestimates the sound diffraction effect on crests, for which the DIN 18005 guideline method is perhaps sufficient.

At last, it appears that it is quite difficult to appreciate and to compare absolute sound immission levels. Moreover, the greater part of studies concern more relative behaviours than absolute levels determination. Therefore, input parameters which modify only the global emission level and consequently, which cause only a uniform increase of sound immission level, require less accuracy in their assessment than those which modify the relative immission field.

These parameters are for example the traffic density, the portion of trucks, the vehicles speed, the road surface material. Although the knowledge of their accurate value would permit to situate the global immission levels, the model user would probably prefer to know how their variation influences that sound level.

\section{Acknowledgements}

The authors would like to thank Mrs. A.C. Romain and Mr. M. Kummert for their kind help when measuring sound levels in the field.

\section{References}

[1] Gabillet Y, Dulau B, Laporte JC, Zouboff JC, Soulage D, Besnard F. Comparison of two methods for predicting traffic noise. Proceedings of Internoise 96, Liverpool, 1996, p. 3133-8.

[2] Petersen J, Kragh J. Testing of software for outdoor noise prediction Proceedings of Internoise 96 -Liverpool, 1996, p. 1863-8.

[3] Pompoli R, Farina A, Fausti P, Bassanino M, Invernizzi S, Menini L. Intercomparison of traffic noise computer simulations. 18th International Congress for Noise Abatement-Traffic Noise in Europe, Association Internationale Contre le Bruit (AICB), Engineering Faculty, 11-13 September 1995, Bologna, 1995.

[4] Van den Berg M. Results of the round robin test on noise calculation models for traffic noise. 18th International Congress for Noise Abatement-Traffic Noise in Europe, Association Internationale Contre le Bruit (AICB), Engineering Faculty, 11-13 September 1995, Bologna, 1995.

[5] Fausti P, Pompoli R. Intercomparison of computer programs for traffic noise simulations Proceedings of Internoise 96, Liverpool, 1996, p. 3143-8.

[6] Van Leeuwen JJA, Manvell D, Nota R. Some prediction models for the calculation of traffic noise in the environment. Proceedings of Internoise 96, Liverpool, 1996, p. 3139-42. 
[7] Din 18005. Teil 1 : Schallschutz im Städtebau-Berechnungsverfahren, Nomenausschu $\beta$ Bauwesen im DIN Deutsches Institut für Normung e.V./Normenausschuß Akustik und Schwingungstechnik im DIN. Berlin: Beuth Verlag GmbH, 1987.

[8] VDI 2714. Schallausbreitung im Freien, VDI-Kommission Lärmminderung. Düsseldorf/Berlin: VDI-Verlag GmbH/Beuth Verlag GmbH, 1988.

[9] RLS-90. Richtlinien für den Lärmschutz an Straßen. Bonn: Der Bundesminister für Verkehr, Abteilung Straßenbau, 1990.

[10] IMMI. Version 4.03 E - User Manual. Höchberg: Wölfel Meßsysteme Software GmbH \& Co, 1995. [11] Migneron JG. Acoustique urbaine MASSON, Paris, 1980, p. 427.

[12] Hassall JR, Zaveri K. In: Brüel and Kjaer, Acoustic noise measurements. Glostrup, p. 310. 